\title{
A New Approximation Method for Common Fixed Points of a Finite Family of Generalized Asymptotically Quasinonexpansive Mappings in Banach Spaces
}

\author{
Pornsak Yatakoat and Suthep Suantai \\ Department of Mathematics, Faculty of Science, Chiangmai University, Chiangmai 50200, Thailand
}

Correspondence should be addressed to Suthep Suantai, scmti005@chiangmai.ac.th

Received 24 June 2010; Accepted 6 September 2010

Academic Editor: Nikolaos Papageorgiou

Copyright (C) 2010 P. Yatakoat and S. Suantai. This is an open access article distributed under the Creative Commons Attribution License, which permits unrestricted use, distribution, and reproduction in any medium, provided the original work is properly cited.

We introduce a new iterative scheme to approximate a common fixed point for a finite family of generalized asymptotically quasinonexpansive mappings. Several strong and weak convergence theorems of the proposed iteration in Banach spaces are established. The main results obtianed in this paper generalize and refine many known results in the current literature.

\section{Introduction}

Let $C$ be a convex subset of a Banach space $X$, and let $\left\{T_{i}: i=1,2, \ldots, k\right\}$ be a family of self-mappings of $C$. Suppose that $\alpha_{i n} \in[0,1]$, for all $n=1,2,3, \ldots$ and $i=1,2, \ldots, k$.

For $x_{1} \in C$, let $\left\{x_{n}\right\}$ be the sequence generated by the following algorithm:

$$
\begin{aligned}
x_{n+1}= & \left(1-\alpha_{k n}\right) x_{n}+\alpha_{k n} T_{k}^{n} y_{(k-1) n} \\
y_{(k-1) n}= & \left(1-\alpha_{(k-1) n}\right) x_{n}+\alpha_{(k-1) n} T_{k-1}^{n} y_{(k-2) n}, \\
y_{(k-2) n}= & \left(1-\alpha_{(k-2) n}\right) x_{n}+\alpha_{(k-2) n} T_{k-2}^{n} y_{(k-3) n}, \\
& \vdots \\
y_{2 n}= & \left(1-\alpha_{2 n}\right) x_{n}+\alpha_{2 n} T_{2}^{n} y_{1 n}, \\
y_{1 n}= & \left(1-\alpha_{1 n}\right) x_{n}+\alpha_{1 n} T_{1}^{n} y_{0 n}
\end{aligned}
$$


where $y_{0 n}=x_{n}$ for all $n$. The iterative process (1.1) for a finite family of mappings introduced by Khan et al. [1], and the iterative process is the generalized form of the modified Mann (one-step) iterative process by Schu [2], the modified Ishikawa (two-step) iterative process by Tan and $\mathrm{Xu}$ [3], and the three-step iterative process by $\mathrm{Xu}$ and Noor [4].

Common fixed points of nonlinear mappings play an important role in solving systems of equations and inequalities. Many researchers [1,5-19] are interested in studying approximation method for finding common fixed points of nonlinear mapping. Also, approximation methods for finding fixed points for nonexpansive mappings can be seen in [12-16, 20, 21].

In 2003, Sun [17] studied an implicit iterative scheme initiated by $\mathrm{Xu}$ and Ori [22] for a finite family of asymptotically quasinonexpansive mappings. Shahzand and Udomene [18], in 2006, proved some convergence theorems for the modified Ishikawa iterative process of two asymptotically quasinonexpence mappings to a common fixed point. Nammanee et al. [23] introduced a three-step iteration scheme for asymptotically nonexpansive mappings and proved weak and strong convergence theorems of that iteration scheme under some control conditions. In 2007, Fukhar-ud-din and Khan [24] studied a new three-step iteration scheme for approximating a common fixed point of asymptotically nonexpansive mappings in uniformly convex Banach spaces. Shahzad and Zegeye [19] introduced a new concept of generalized asymptotially nonexpansive mappings and proved some strong convergence theorems for fixed points of finite family of this class. Recently, Khan et al. [1] introduced the iterative sequence (1.1) for a finite family of asymptotically quasinonexpansive mappings in Banach spaces.

Motivated by Khan et al. [1], we introduce a new iterative scheme for finding a common fixed point of a finite family of generalized asymptotically quasinonexpansive mappings as follows:

For $x_{1} \in C$, let $\left\{x_{n}\right\}$ be the sequence generated by

$$
\begin{aligned}
x_{n+1}= & \left(1-\alpha_{k n}\right) y_{(k-1) n}+\alpha_{k n} T_{k}^{n} y_{(k-1) n}, \\
y_{(k-1) n}= & \left(1-\alpha_{(k-1) n}\right) y_{(k-2) n}+\alpha_{(k-1) n} T_{k-1}^{n} y_{(k-2) n}, \\
y_{(k-2) n}= & \left(1-\alpha_{(k-2) n}\right) y_{(k-3) n}+\alpha_{(k-2) n} T_{k-2}^{n} y_{(k-3) n} \\
& \vdots \\
y_{2 n}= & \left(1-\alpha_{2 n}\right) y_{1 n}+\alpha_{2 n} T_{2}^{n} y_{1 n}, \\
y_{1 n}= & \left(1-\alpha_{1 n}\right) y_{0 n}+\alpha_{1 n} T_{1}^{n} y_{0 n},
\end{aligned}
$$

where $y_{0 n}=x_{n}$ for all $n$.

The aim of this paper is to obtain strong and weak convergence results for the iterative process (1.2) of a finite family of generalized asymptotically quasinonexpansive mappings in Banach spaces.

\section{Preliminaries}

In this section, we give some definitions and lemmas used in the main results. 
Let $C$ be a nonempty subset of a real Banach space $X$, and let $T$ be a self-mapping of $C$. The fixed point set of $T$ is denoted by $F(T)=\{x \in C: T x=x\}$.

Then let $T$ is called

(i) nonexpansive if $\|T x-T y\| \leq\|x-y\|$, for all $x, y \in C$;

(ii) quasinonexpansive if $F(T) \neq \emptyset$ and $\|T x-p\| \leq\|x-p\|$, for all $x \in C$ and $p \in F(T)$;

(iii) asymptotically nonexpansive if there exists a sequence $\left\{r_{n}\right\}$ in $[0, \infty)$ with $\lim _{n \rightarrow \infty} r_{n}=$ 0 and $\left\|T^{n} x-T^{n} y\right\| \leq\left(1+r_{n}\right)\|x-y\|$, for all $x, y \in C$ and $n=1,2,3, \ldots$;

(iv) asymptotically quasinonexpansive if $F(T) \neq \emptyset$ and there exists a sequence $\left\{r_{n}\right\}$ in $[0, \infty)$ with $\lim _{n \rightarrow \infty} r_{n}=0$ and $\left\|T^{n} x-p\right\| \leq\left(1+r_{n}\right)\|x-p\|$, for all $x \in C, p \in F(T)$ and $n=1,2,3, \ldots$;

(v) generalized quasinonexpansive if $F(T) \neq \emptyset$ and there exists a sequence $\left\{s_{n}\right\}$ in $[0, \infty)$ with $s_{n} \rightarrow 0$ as $n \rightarrow \infty$ such that $\left\|T^{n} x-p\right\| \leq\|x-p\|+s_{n}$, for all $x \in C, p \in F(T)$ and $n=1,2,3, \ldots$;

(vi) generalized asymptotically quasinonexpansive [19] if $F(T) \neq \emptyset$ and there exist two sequences $\left\{r_{n}\right\}$ and $\left\{s_{n}\right\}$ in $[0, \infty)$ with $r_{n} \rightarrow 0$ and $s_{n} \rightarrow 0$ as $n \rightarrow \infty$ such that $\left\|T^{n} x-p\right\| \leq\left(1+r_{n}\right)\|x-p\|+s_{n}$, for all $x \in C, p \in F(T)$ and $n=1,2,3, \ldots ;$

(vii) uniformly L-Lipschitzian if there exists constant $L>0$ such that $\left\|T^{n} x-T^{n} y\right\| \leq$ $L\|x-y\|$, for all $x, y \in C$ and $n=1,2,3, \ldots$;

(viii) $(L-\gamma)$ uniform Lipschitz if there are constants $L>0$ and $\gamma>0$ such that $\left\|T^{n} x-T^{n} y\right\| \leq$ $L\|x-y\|^{\gamma}$, for all $x, y \in C$ and $n=1,2,3, \ldots$.

(ix) semicompact if for a sequence $\left\{x_{n}\right\}$ in $C$ with $\lim _{n \rightarrow \infty}\left\|x_{n}-T x_{n}\right\|=0$, there exists a subsequence $\left\{x_{n_{i}}\right\}$ of $\left\{x_{n}\right\}$ such that $x_{n_{i}} \rightarrow p \in C$.

From the definition of these mappings, it can be seen that

(i) a quasinonexpansive mapping is generalized quasinonexpansive;

(ii) an asymptotically quasinonexpansive mapping is generalized asymptotically quasinonexpansive;

(iii) a generalized quasinonexpansive mapping is generalized asymptotically quasinonexpansive;

(iv) a uniformly L-Lipschitzian mapping is $(L-1)$ uniform Lipschitz.

The map $T: C \rightarrow X$ is said to be demiclosed at 0 if for each sequence $\left\{x_{n}\right\}$ in $C$ converging weakly to $x \in C$ and $T\left(x_{n}\right)$ converging strongly to 0 , we get $T x=0$.

A Banach space $X$ is said to have Opial's property if for each sequence $\left\{x_{n}\right\}$ converging weakly to $x \in C$ and $x \neq y$, we have the condition

$$
\liminf _{n \rightarrow \infty}\left\|x_{n}-x\right\|<\liminf _{n \rightarrow \infty}\left\|x_{n}-y\right\|
$$

Condition $\left(\mathrm{A}^{\prime \prime}\right)$. Let $C$ be a subset of a normed space $X$. A family of self-mappings $\left\{T_{i}: i=\right.$ $1,2, \ldots, k\}$ of $C$ is said to have Condition $\left(\mathrm{A}^{\prime \prime}\right)$ if there exists a nondecreasing function $f$ : $[0, \infty) \rightarrow[0, \infty)$ with $f(0)=0$ and $f(r)>0$ for all $r \in(0, \infty)$ such that $\left\|x-T_{i} x\right\| \geq f(d(x, F))$ for some $1 \leq i \leq k$ and for all $x \in C$ where $d(x, F)=\inf \left\{\|x-p\|: p \in F=\bigcap_{i=1}^{k} F\left(T_{i}\right)\right\}$. 
The condition $\left(\mathrm{A}^{\prime \prime}\right)$ defined above by the authors is the generalization of the condition (A) [25] when $k=1$ and condition ( $\left.\mathrm{A}^{\prime}\right)$ [26] for $k=2$.

The following lemmas are needed for proving our main results.

Lemma 2.1 (cf. [17, Lemma 2.2]). Let the sequences $\left\{a_{n}\right\},\left\{\delta_{n}\right\}$ and $\left\{c_{n}\right\}$ of real numbers satisfy:

$$
a_{n+1} \leq\left(1+\delta_{n}\right) a_{n}+c_{n}, \text { where } a_{n} \geq 0, \delta_{n} \geq 0, c_{n} \geq 0 \forall n=1,2,3, \ldots
$$

and $\sum_{n=1}^{\infty}, \delta_{n}<\infty, \sum_{n=1}^{\infty}, c_{n}<\infty$.

(i) $\lim _{n \rightarrow \infty} a_{n}$ exists;

(ii) if $\lim \inf _{n \rightarrow \infty} a_{n}=0$, then $\lim _{n \rightarrow \infty} a_{n}=0$.

Lemma 2.2 (see [2, Lemma 1.3]). Let $X$ be a uniformly convex Banach space. Assume that $0<b \leq$ $t_{n} \leq c<1, n=1,2,3, \ldots$. Let the sequences $\left\{x_{n}\right\}$ and $\left\{y_{n}\right\}$ in $X$ be such that $\limsup _{n \rightarrow \infty}\left\|x_{n}\right\| \leq a$, $\limsup \sup _{n \rightarrow \infty}\left\|y_{n}\right\| \leq$ a and $\lim _{n \rightarrow \infty}\left\|t_{n} x_{n}+\left(1-t_{n}\right) y_{n}\right\|=a$, where $a \geq 0$. Then $\lim _{n \rightarrow \infty}\left\|x_{n}-y_{n}\right\|=0$.

\section{Convergence in Banach Spaces}

The aim of this section is to establish the strong convergence of the iterative scheme (1.2) to converge to a common fixed point of a finite family of asymptotically quasinonexpansive mappings in a Banach space under some appropriate conditions.

Lemma 3.1. Let $C$ be a nonempty closed convex subset of a real Banach space $X$, and $\left\{T_{i}: i=\right.$ $1,2, \ldots, k\}$ be a finite family of generalized asymtotically quasinonexpansive self-mappings of $C$, that is, $\left\|T_{i}^{n} x-p_{i}\right\| \leq\left(1+r_{i n}\right)\left\|x-p_{i}\right\|+s_{i n}$, for all $x \in C$ with the sequence $\left\{r_{i n}\right\},\left\{s_{i n}\right\} \subset[0, \infty)$ and $p_{i} \in F\left(T_{i}\right), i=1,2, \ldots, k$. Suppose that $F=\bigcap_{i=1}^{k} F\left(T_{i}\right) \neq \emptyset, x_{1} \in C$, and the iterative sequence $\left\{x_{n}\right\}$ is defined by (1.2). Let $r_{n}=\max _{1 \leq i \leq k}\left\{r_{i n}\right\}$ and $s_{n}=\max _{1 \leq i \leq k}\left\{s_{i n}\right\}$ Then for $p \in F$, we get the following:

(i) $\left\|x_{n}-T_{i}^{n} x_{n}\right\| \leq\left(2+r_{n}\right)\left\|x_{n}-p\right\|+s_{n}$, for all $i=1,2, \ldots, k$;

(ii) $\left\|y_{(i-1) n}-T_{i}^{n} y_{(i-1) n}\right\| \leq\left(2+r_{n}\right)\left\|y_{(i-1) n}-p\right\|+s_{n}$, for all $i=1,2, \ldots, k$;

(iii) $\left\|y_{k n}-p\right\| \leq\left(1+r_{n}\right)^{k}\left\|x_{n}-p\right\|+s_{n} \sum_{i=1}^{k}\left(1+r_{n}\right)^{k-i}$;

(iv) $\left\|x_{n+1}-p\right\| \leq\left(1+r_{n}\right)^{k}\left\|x_{n}-p\right\|+s_{n} \sum_{i=1}^{k}\left(1+r_{n}\right)^{(k-i)}$;

(v) $\left\|x_{n+1}-p\right\| \leq\left(1+\delta_{n}\right)\left\|x_{n}-p\right\|+c_{n}$, for all $n \in \mathbb{N}$, where $c_{n}=s_{n} \sum_{i=1}^{k}\left(1+r_{n}\right)^{(k-i)}$ and $\delta_{n}=\left(\begin{array}{c}k \\ 1\end{array}\right) r_{n}+\left(\begin{array}{c}k \\ 2\end{array}\right) r_{n}^{2}+\cdots+\left(\begin{array}{c}k \\ k\end{array}\right) r_{n}^{k}$.

(vi) If $\sum_{n=1}^{\infty} r_{n}<\infty$ and $\sum_{n=1}^{\infty} s_{n}<\infty$, then $\lim _{n \rightarrow \infty}\left\|x_{n}-p\right\|$ exists.

Proof. Let $p \in F$.

(i) For $i=1,2,3, \ldots, k$, we have

$$
\begin{aligned}
\left\|x_{n}-T_{i}^{n} x_{n}\right\| & \leq\left\|x_{n}-p\right\|+\left\|T_{i}^{n} x_{n}-p\right\| \\
& \leq\left\|x_{n}-p\right\|+\left(1+r_{n}\right)\left\|x_{n}-p\right\|+s_{i n} \\
& \leq\left(2+r_{n}\right)\left\|x_{n}-p\right\|+s_{n} .
\end{aligned}
$$


(ii) Similarly to part (i), we have

$$
\left\|y_{(i-1) n}-T_{i}^{n} y_{(i-1) n}\right\| \leq\left(2+r_{n}\right)\left\|y_{(i-1) n}-p\right\|+s_{n}, \quad \forall i=1,2, \ldots, k
$$

(iii) By part (i) and $0 \leq \alpha_{\text {in }} \leq 1$, we have

$$
\begin{aligned}
\left\|y_{1 n}-p\right\| & =\left\|\left(1-\alpha_{1 n}\right)\left(x_{n}-p\right)+\alpha_{1 n}\left(T_{1}^{n} x_{n}-p\right)\right\| \\
& \leq\left(1-\alpha_{1 n}\right)\left\|x_{n}-p\right\|+\alpha_{1 n}\left\|T_{1}^{n} x_{n}-p\right\| \\
& \leq\left(1-\alpha_{1 n}\right)\left\|x_{n}-p\right\|+\alpha_{1 n}\left(1+r_{1 n}\right)\left\|x_{n}-p\right\|+\alpha_{1 n} s_{1 n} \\
& \leq\left(1+r_{n}\right)\left\|x_{n}-p\right\|+s_{n} \\
\left\|y_{2 n}-p\right\|= & \left\|\left(1-\alpha_{2 n}\right)\left(y_{1 n}-p\right)+\alpha_{2 n}\left(T_{2}^{n} y_{1 n}-p\right)\right\| \\
\leq & \left(1-\alpha_{2 n}\right)\left\|y_{1 n}-p\right\|+\alpha_{2 n}\left\|T_{2}^{n} y_{1 n}-p\right\| \\
\leq & \left(1-\alpha_{2 n}\right)\left\|y_{1 n}-p\right\|+\alpha_{2 n}\left(1+r_{2 n}\right)\left\|y_{1 n}-p\right\|+\alpha_{2 n} s_{2 n} \\
\leq & \left(1+r_{n}\right)\left\|y_{1 n}-p\right\|+s_{n} \\
\leq & \left(1+r_{n}\right)\left(1+r_{n}\right)\left\|x_{n}-p\right\|+s_{n}\left(1+r_{n}\right)+s_{n} \\
\leq & \left(1+r_{n}\right)^{2}\left\|x_{n}-p\right\|+s_{n}\left(\left(1+r_{n}\right)+1\right) \\
\left\|y_{3 n}-p\right\|= & \left\|\left(1-\alpha_{3 n}\right)\left(y_{2 n}-p\right)+\alpha_{3 n}\left(T_{3}^{n} y_{2 n}-p\right)\right\| \\
\leq & \left(1-\alpha_{3 n}\right)\left\|y_{2 n}-p\right\|+\alpha_{3 n}\left\|T_{3}^{n} y_{2 n}-p\right\| \\
\leq & \left(1-\alpha_{3 n}\right)\left\|y_{2 n}-p\right\|+\alpha_{3 n}\left(1+r_{3 n}\right)\left\|y_{2 n}-p\right\|+\alpha_{3 n} s_{3 n} \\
\leq & \left(1+r_{n}\right)\left\|y_{2 n}-p\right\|+s_{n} \\
\leq & \left(1+r_{n}\right)^{3}\left\|x_{n}-p\right\|+s_{n}\left(\left(1+r_{n}\right)^{2}+\left(1+r_{n}\right)+1\right) \\
& \vdots \\
\left\|y_{k n}-p\right\| \leq & \left(1+r_{n}\right)^{k}\left\|x_{n}-p\right\|+s_{n} \sum_{i=1}\left(1+r_{n}\right)^{(k-i)} .
\end{aligned}
$$

(iv) By part (ii) and part (iii), we get

$$
\begin{aligned}
\left\|x_{n+1}-p\right\| & =\left\|\left(1-\alpha_{k n}\right)\left(y_{(k-1) n}-p\right)+\alpha_{k n}\left(T_{k}^{n} y_{(k-1) n}-p\right)\right\| \\
& \leq\left(1-\alpha_{k n}\right)\left\|y_{(k-1) n}-p\right\|+\alpha_{k n}\left\|T_{k}^{n} y_{(k-1) n}-p\right\| \\
& \leq\left(1-\alpha_{k n}\right)\left\|y_{(k-1) n}-p\right\|+\alpha_{k n}\left(1+r_{n}\right)\left\|y_{(k-1) n}-p\right\|+\alpha_{k n} s_{k n} \\
& \leq\left(1+r_{n}\right)\left\|y_{(k-1) n}-p\right\|+s_{n} \\
& \leq\left(1+r_{n}\right)\left(1+r_{n}\right)^{k-1}\left\|x_{n}-p\right\|+\left(1+r_{n}\right) s_{n} \sum_{i=1}^{k-1}\left(1+r_{n}\right)^{(k-1-i)}+s_{n} \\
& =\left(1+r_{n}\right)^{k}\left\|x_{n}-p\right\|+s_{n} \sum_{i=1}^{k}\left(1+r_{n}\right)^{(k-i)} .
\end{aligned}
$$


(v) Put $\delta_{n}=\left(\begin{array}{c}k \\ 1\end{array}\right) r_{n}+\left(\begin{array}{c}k \\ 2\end{array}\right) r_{n}^{2}+\cdots+\left(\begin{array}{c}k \\ k\end{array}\right) r_{n}^{k}$ and $c_{n}=s_{n} \sum_{i=1}^{k}\left(1+r_{n}\right)^{(k-i)}$. Then (v) is directly obtained by (iv).

(vi) By $(v)$, we have $\left\|x_{n+1}-p\right\| \leq\left(1+\delta_{n}\right)\left\|x_{n}-p\right\|+c_{n}$ for all $n \in N$, where $c_{n}=$ $s_{n} \sum_{i=1}^{k}\left(1+r_{n}\right)^{(k-i)}$ and $\delta_{n}=\left(\begin{array}{c}k \\ 1\end{array}\right) r_{n}+\left(\begin{array}{c}k \\ 2\end{array}\right) r_{n}^{2}+\cdots+\left(\begin{array}{c}k \\ k\end{array}\right) r_{n}^{k}$. From $\sum_{n=1}^{\infty} r_{n}<\infty$ and $\sum_{n=1}^{\infty} s_{n}<\infty$, it follows that $\sum_{n=1}^{\infty} \delta_{n}<\infty$ and $\sum_{n=1}^{\infty} c_{n}<\infty$. By Lemma 2.1, we get $\lim _{n \rightarrow \infty}\left\|x_{n}-p\right\|$ exists.

Theorem 3.2. Let $C$ be a nonempty closed convex subset of a real Banach space $X$, and $\left\{T_{i}: i=\right.$ $1,2, \ldots, k\}$ be a finite family of generalized asymtotically quasinonexpansive self-mappings of $C$, that is, $\left\|T_{i}^{n} x-p_{i}\right\| \leq\left(1+r_{i n}\right)\left\|x-p_{i}\right\|+s_{i n}$, for all $x \in C$ and $p_{i} \in F\left(T_{i}\right), i=1,2, \ldots, k$. Suppose that $F=\bigcap_{i=1}^{k} F\left(T_{i}\right) \neq \emptyset$ is closed, $x_{1} \in C$ and the iterative sequence $\left\{x_{n}\right\}$ is defined by (1.2). Assume that $\sum_{n=1}^{\infty} r_{n}<\infty$ and $\sum_{n=1}^{\infty} s_{n}<\infty$, where $r_{n}=\max _{1 \leq i \leq k}\left\{r_{i n}\right\}$ and $s_{n}=\max _{1 \leq i \leq k}\left\{s_{i n}\right\}$. Then $\left\{x_{n}\right\}$ converges strongly to a common fixed point of the family of mappings if and only if $\liminf _{n \rightarrow \infty} d\left(x_{n}, F\right)=0$, where $d(x, F)=\inf _{p \in F}\|x-p\|$.

Proof. The necessity is obvious and then we prove only the sufficiency. Let $p \in F$. Since $1+t \leq$ $e^{t}$ for $t \geq 0$, we obtain $(1+t)^{k} \leq e^{k t}$, for $k=1,2, \ldots$ Thus by Lemma 3.1(iv) and (v), for positive integers $m$ and $n$, we have

$$
\begin{aligned}
\left\|x_{n+m}-p\right\| & \leq\left(1+r_{(n+m-1)}\right)^{k}\left\|x_{(n+m-1)}-p\right\|+c_{(n+m-1)} \\
& \leq \exp \left\{k r_{(n+m-1)}\right\}\left\|x_{(n+m-1)}-p\right\|+c_{(n+m-1)} \\
& \leq \cdots \leq \exp \left\{k \sum_{i=n}^{n+m-1} r_{i}\right\}\left\|x_{n}-p\right\|+\sum_{i=n}^{n+m-1} c_{i} \\
& \leq \exp \left\{k \sum_{i=1}^{\infty} r_{i}\right\}\left\|x_{n}-p\right\|+\sum_{i=n}^{\infty} c_{i} \\
& =M\left\|x_{n}-p\right\|+\sum_{i=n}^{\infty} c_{i},
\end{aligned}
$$

where $M=\exp \left\{k \sum_{i=1}^{\infty} r_{i}\right\}$

By Lemma 3.1(v), we have

$$
\left\|x_{n+1}-p\right\| \leq\left(1+\delta_{n}\right)\left\|x_{n}-p\right\|+c_{n}, \quad \forall p \in F,
$$

where $\delta_{n}=\left(\begin{array}{c}k \\ 1\end{array}\right) r_{n}+\left(\begin{array}{c}k \\ 2\end{array}\right) r_{n}^{2}+\cdots+\left(\begin{array}{c}k \\ k\end{array}\right) r_{n}^{k}$ and $c_{n}=s_{n} \sum_{i=1}^{k}\left(1+r_{n}\right)^{(k-i)}$.

It follows that

$$
d\left(x_{n+1}, F\right) \leq\left(1+\delta_{n}\right) d\left(x_{n}, F\right)
$$

From the given condition $\lim \inf _{n \rightarrow \infty} d\left(x_{n}, F\right)=0$ and Lemma 2.1, we get

$$
\lim _{n \rightarrow \infty} d\left(x_{n}, F\right)=0
$$


Next, we show that $\left\{x_{n}\right\}$ is a Cauchy sequence in C. By (3.8) and $\sum_{n=1}^{\infty} c_{n}<\infty$, we get that for any $\epsilon>0$, there exists a positive integer $n_{0}$ such that, for all $n \geq n_{0}$,

$$
d\left(x_{n}, F\right)<\frac{\epsilon}{3 M}, \quad \sum_{n=n_{0}}^{\infty} c_{n}<\frac{\epsilon}{3}
$$

From $d\left(x_{n}, F\right)<\epsilon / 3 M$, there exists $p_{0} \in F$ such that

$$
\left\|x_{n_{0}}-p_{0}\right\|<\frac{\epsilon}{3 M}
$$

For any positive integer $m$, by (3.5), (3.9), and (3.10), we have

$$
\begin{aligned}
\left\|x_{n_{0}+m}-x_{n_{0}}\right\| & \leq\left\|x_{n_{0}+m}-p_{0}\right\|+\left\|x_{n_{0}}-p_{0}\right\| \\
& \leq M\left\|x_{n_{0}}-p_{0}\right\|+\sum_{i=n_{0}}^{\infty} c_{i}+\left\|x_{n_{0}}-p_{0}\right\| \\
& <M \frac{\epsilon}{3 M}+\frac{\epsilon}{3}+M \frac{\epsilon}{3 M}=\epsilon .
\end{aligned}
$$

Thus, $\left\{x_{n}\right\}$ is a Cauchy sequence in $X$. Since $X$ is complete, $x_{n} \rightarrow q \in X$. Actually, $q \in C$ because $\left\{x_{n}\right\} \subset C$ and $C$ is a closed subset of $X$. Next we show that $q \in F$. Since $F=\bigcap_{i=1}^{k} F\left(T_{i}\right)$ is closed, by the continuity of $d(x, F)$ with $d\left(x_{n}, F\right) \rightarrow 0$ and $x_{n} \rightarrow q$ as $n \rightarrow \infty$, we get $d(q, F)=0$ and then $q \in F$. Therefore, the proof is complete.

Since any asymptotically quasinonexpansive mapping is generalized asymptotically quasinonexpansive, the next corollary is obtained immediately from Theorem 3.2.

Corollary 3.3 (see [5, Theorem 3.2]). Let $C$ be a nonempty closed convex subset of a real Banach space $X$, and $\left\{T_{i}: i=1,2, \ldots, k\right\}$ be a finite family of asymptotically quasinonexpansive self-mappings of $C$, that is, $\left\|T_{i}^{n} x-p_{i}\right\| \leq\left(1+r_{i n}\right)\left\|x-p_{i}\right\|$, for all $x \in C$ and $p_{i} \in F\left(T_{i}\right), i=1,2, \ldots, k . x_{1} \in C$ and the iterative sequence $\left\{x_{n}\right\}$ be defined by (1.2). Then $\left\{x_{n}\right\}$ converges strongly to a common fixed point of the family of mappings if and only if $\lim _{\inf _{n \rightarrow \infty}} d\left(x_{n}, F\right)=0$, where $d(x, F)=\inf _{p \in F}\|x-p\|$.

\section{Convergence in Uniformly Convex Banach Spaces}

In this section, strong and weak convergence results for the iterative process (1.2) on uniformly convex Banach spaces are proved without using the condition lim $\inf _{n \rightarrow \infty} d\left(x_{n}, F\right)=0$ appearing in Section 3.

Theorem 4.1. Let $C$ be a nonempty closed convex subset of an uniformly convex real Banach space $X$. Let $\left\{T_{i}: i=1,2, \ldots, k\right\}$ be a finite family of uniformly $\left(L-\gamma_{i}\right)$ Lipschitzian and generalized asymptotically quasinonexpansive self-mappings of $C$, that is, $\left\|T_{i}^{n} x-T_{i}^{n} y\right\| \leq L\|x-y\|^{\gamma_{i}}$ and $\| T_{i}^{n} x-$ $p_{i}\left\|\leq\left(1+r_{i n}\right)\right\| x-p_{i} \|+s_{i n}$, for all $x, y \in C$ and $p_{i} \in F\left(T_{i}\right), i=1,2, \ldots, k$. Suppose that $\left\{T_{i}: i=\right.$ $1,2, \ldots, k\}$ satisfies condition $\left(A^{\prime \prime}\right)$ and $F=\bigcap_{i=1}^{k} F\left(T_{i}\right) \neq \emptyset$. Let $x_{1} \in C$ and the iterative sequence $\left\{x_{n}\right\}$ be defined by (1.2) with $\left\{\alpha_{i n}\right\}_{i=1}^{n} \subset[a, b]$, where $0<a<b<1$. Assume that $\sum_{n=1}^{\infty} r_{n}<\infty$, 
$\sum_{n=1}^{\infty} s_{n}<\infty$ where $r_{n}=\max _{1 \leq i \leq k}\left\{r_{i n}\right\}$ and $s_{n}=\max _{1 \leq i \leq k}\left\{s_{i n}\right\}$. Then $\left\{x_{n}\right\}$ converges strongly to $a$ common fixed point of the family of mappings.

Proof. Let $p \in$ F. By Lemma 3.1(vi), we get that $\lim _{n \rightarrow \infty}\left\|x_{n}-p\right\|$ exists. Then there is a real number $c \geq 0$ such that

$$
\lim _{n \rightarrow \infty}\left\|x_{n}-p\right\|=c
$$

By Lemma 3.1(iii), we have

$$
\left\|y_{k n}-p\right\| \leq\left(1+r_{n}\right)^{k}\left\|x_{n}-p\right\|+s_{n} \sum_{i=1}^{k}\left(1+r_{n}\right)^{(k-i)}, \quad \forall p \in F
$$

By taking lim sup on both sides of the above inequality, we get

$$
\limsup _{n \rightarrow \infty}\left\|y_{\text {in }}-p\right\| \leq c, \quad \text { for } i=1,2, \ldots, k-1 .
$$

Since $\left\|T_{i}^{n} y_{(i-1) n}-p\right\| \leq\left(1+r_{n}\right)\left\|y_{(i-1) n}-p\right\|+s_{n}$ and (4.3), we obtain

$$
\limsup _{n \rightarrow \infty}\left\|T_{i}^{n} y_{(i-1) n}-p\right\| \leq c, \quad \text { for } i=1,2, \ldots, k
$$

Since $\lim _{n \rightarrow \infty}\left\|x_{n+1}-p\right\|=c$, we have

$$
\lim _{n \rightarrow \infty}\left\|\left(1-\alpha_{k n}\right)\left(y_{(k-1) n}-p\right)+\alpha_{k n}\left(T_{k}^{n} y_{(k-1) n}-p\right)\right\|=c
$$

Using (4.1), (4.4), and Lemma 2.2, we conclude that

$$
\lim _{n \rightarrow \infty}\left\|y_{(k-1) n}-T_{k}^{n} y_{(k-1) n}\right\|=0
$$

We assume that

$$
\lim _{n \rightarrow \infty}\left\|y_{(j-1) n}-T_{j}^{n} y_{(j-1) n}\right\|=0, \quad \text { for some } 2 \leq j \leq k
$$

It follows from (4.5) and (4.7) that

$$
c \leq \liminf _{n \rightarrow \infty}\left\|y_{(j-1) n}-p\right\|, \quad \text { for } 2 \leq j \leq k .
$$

By Lemma 3.1(iv), (1.2), and (4.8), we get

$$
\lim _{n \rightarrow \infty}\left\|\left(1-\alpha_{(j-1) n}\right)\left(y_{(j-2) n}-p\right)+\alpha_{(j-1) n}\left(T_{j-1}^{n} y_{(j-2) n}-p\right)\right\|=\lim _{n \rightarrow \infty}\left\|y_{(j-1) n}-p\right\|=c
$$


Using (4.3), (4.4) and Lemma 2.2, we conclude that

$$
\lim _{n \rightarrow \infty}\left\|y_{(j-2) n}-T_{j-1}^{n} y_{(j-2) n}\right\|=0
$$

Therefore, by mathematical induction, we obtain

$$
\lim _{n \rightarrow \infty}\left\|y_{(i-1) n}-T_{i}^{n} y_{(i-1) n}\right\|=0, \quad \text { for } i=1,2, \ldots, k
$$

From (1.2), we have

$$
\left\|y_{i n}-y_{(i-1) n}\right\|=\alpha_{i n}\left\|T_{i}^{n} y_{(i-1) n}-y_{(j-1) n}\right\|, \quad \text { for } i=1,2, \ldots, k-1
$$

By (4.11), we obtain that

$$
\left\|y_{i n}-y_{(i-1) n}\right\| \longrightarrow 0 \text { as } n \longrightarrow \infty, \text { for } i=1,2, \ldots, k-1
$$

From

$$
\left\|x_{n}-y_{i n}\right\| \leq\left\|x_{n}-y_{1 n}\right\|+\left\|y_{1 n}-y_{2 n}\right\|+\cdots+\left\|y_{(i-1) n}-y_{i n}\right\|,
$$

for $i=1,2, \ldots, k-1$, it follows by (4.13) that

$$
\left\|x_{n}-y_{\text {in }}\right\| \longrightarrow 0 \text { as } n \longrightarrow \infty, \text { for } i=1,2, \ldots, k-1
$$

From (4.11), when $i=1$, we get $\lim _{n \rightarrow \infty}\left\|x_{n}-T_{1}^{n} x_{n}\right\|=0$. For $2 \leq i \leq k$, we have

$$
\begin{aligned}
\left\|x_{n}-T_{i}^{n} x_{n}\right\| & \leq\left\|x_{n}-y_{(i-1) n}\right\|+\left\|y_{(i-1) n}-T_{i}^{n} y_{(i-1) n}\right\|+\left\|T_{i}^{n} y_{(i-1) n}-T_{i}^{n} x_{n}\right\| \\
& \leq\left\|x_{n}-y_{(i-1) n}\right\|+\left\|y_{(i-1) n}-T_{i}^{n} y_{(i-1) n}\right\|+L\left\|y_{(i-1) n}-x_{n}\right\|^{\gamma_{i}} .
\end{aligned}
$$

From (4.11) and (4.15), we conclude that

$$
\lim _{n \rightarrow \infty} \gamma_{i n}=0, \quad \text { for } i=1,2, \ldots, k
$$

where $\gamma_{i n}=\left\|x_{n}-T_{i}^{n} x_{n}\right\|$. From (1.2), we have

$$
\begin{aligned}
\left\|x_{n+1}-x_{n}\right\| & \leq\left(1-\alpha_{k n}\right)\left\|y_{(k-1) n}-x_{n}\right\|+\alpha_{k n}\left\|T_{k}^{n} y_{(k-1) n}-x_{n}\right\| \\
& \leq\left(1-\alpha_{k n}\right)\left\|y_{(k-1) n}-x_{n}\right\|+\alpha_{k n}\left(\left\|T_{k}^{n} y_{(k-1) n}-y_{(k-1) n}\right\|+\left\|y_{(k-1) n}-x_{n}\right\|\right) \\
& =\left\|y_{(k-1) n}-x_{n}\right\|+\alpha_{k n}\left\|T_{k}^{n} y_{(k-1) n}-y_{(k-1) n}\right\| .
\end{aligned}
$$


From (4.11) and (4.15),

$$
\lim _{n \rightarrow \infty}\left\|x_{n+1}-x_{n}\right\|=0
$$

For $i=1,2, \ldots, k$, we have

$$
\begin{aligned}
\left\|x_{n+1}-T_{i} x_{n+1}\right\| & \leq\left\|x_{n+1}-T_{i}^{n+1} x_{n+1}\right\|+\left\|T_{i} x_{n+1}-T_{i}^{n+1} x_{n+1}\right\| \\
& \leq \gamma_{i(n+1)}+L\left\|x_{n+1}-T_{i}^{n} x_{n+1}\right\|^{\gamma_{i}} \\
& \leq \gamma_{i(n+1)}+L\left(\left\|x_{n+1}-x_{n}\right\|+\left\|x_{n}-T_{i}^{n} x_{n}\right\|+\left\|T_{i}^{n} x_{n}-T_{i}^{n} x_{n+1}\right\|\right)^{\gamma_{i}} \\
& \leq \gamma_{i(n+1)}+L\left(\left\|x_{n+1}-x_{n}\right\|+\gamma_{i n}+L\left\|x_{n}-x_{n+1}\right\|^{\gamma_{i}}\right)^{\gamma_{i}} .
\end{aligned}
$$

Using (4.17) and (4.19), we obtain

$$
\lim _{n \rightarrow \infty}\left\|x_{n+1}-T_{i} x_{n+1}\right\|=0, \quad \text { for } i=1,2, \ldots, k
$$

Therefore, by using condition $\left(\mathrm{A}^{\prime \prime}\right)$, there exists a nondecreasing function $f:[0, \infty) \rightarrow[0, \infty)$ with $f(0)=0$ and $f(r)>0$ for all $r \in(0, \infty)$ such that

$$
\lim _{n \rightarrow \infty} f\left(d\left(x_{n}, F\right)\right) \leq \lim _{n \rightarrow \infty}\left\|x_{n}-T_{j} x_{n}\right\|=0,
$$

for some $1 \leq j \leq k$, that is

$$
\lim _{n \rightarrow \infty} d\left(x_{n}, F\right)=0
$$

By Theorem 3.2, we conclude that $\left\{x_{n}\right\}$ converges strongly to a point $p \in F$.

Lemma 4.2. Let $C$ be a nonempty closed convex subset of an uniformly convex real Banach space $X$, and $\left\{T_{i}: i=1,2, \ldots, k\right\}$ be a family of $\left(L-\gamma_{i}\right)$ uniform Lipschitz and generalized asymtotically quasinonexpansive self-mappings of $C$, that is, $\left\|T_{i}^{n} x-T_{i}^{n} y\right\| \leq L\|x-y\|^{\gamma_{i}}$ and $\left\|T_{i}^{n} x-p_{i}\right\| \leq(1+$ $\left.r_{i n}\right)\left\|x-p_{i}\right\|+s_{i n}$, for all $x, y \in C$ and $p_{i} \in F\left(T_{i}\right), i=1,2, \ldots, k$. Suppose that $F=\bigcap_{i=1}^{k} F\left(T_{i}\right) \neq \emptyset$. Let $x_{1} \in C$ and the iterative sequence $\left\{x_{n}\right\}$ be defined by (1.2) with $\left\{\alpha_{i n}\right\}_{i=1}^{n} \subset[a, b]$, where $0<a<b<1$. Assume that $\sum_{n=1}^{\infty} r_{n}<\infty, \sum_{n=1}^{\infty} s_{n}<\infty$ where $r_{n}=\max _{1 \leq i \leq k}\left\{r_{i n}\right\}$ and $s_{n}=\max _{1 \leq i \leq k}\left\{s_{i n}\right\}$. Then,

(i) $\lim _{n \rightarrow \infty}\left\|x_{n}-T_{i}^{n} y_{(i-1) n}\right\|=0$, for all $i=1,2, \ldots, k$;

(ii) $\lim _{n \rightarrow \infty}\left\|x_{n}-T_{i} x_{n}\right\|=0$, for all $i=1,2, \ldots, k$.

Proof. (i) Let $p \in F$. By Lemma 3.1(vi), we obtain that $\lim _{n \rightarrow \infty}\left\|x_{n}-p\right\|$ exists and we then suppose that

$$
\lim _{n \rightarrow \infty}\left\|x_{n}-p\right\|=c .
$$


By (4.24) and Lemma 3.1(iii), we have

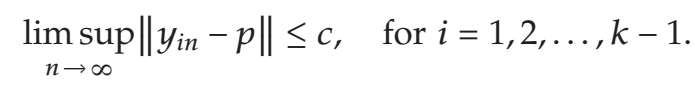

By (1.2), we have

$$
\begin{aligned}
\left\|x_{n+1}-p\right\| \leq & \left(1-\alpha_{k n}\right)\left\|y_{(k-1) n}-p\right\|+\alpha_{k n}\left\|T_{k}^{n} y_{(k-1) n}-p\right\| \\
& \leq\left(1-\alpha_{k n}\right)\left\|y_{(k-1) n}-p\right\|+\alpha_{k n}\left(1+r_{n}\right)\left\|y_{(k-1) n}-p\right\|+\alpha_{k n} s_{k n} \\
& \leq\left(1+r_{n}\right)\left\|y_{(k-1) n}-p\right\|+s_{n} \\
& =\left(1+r_{n}\right)\left\|\left(1-\alpha_{(k-1) n}\right)\left(y_{(k-2) n}-p\right)+\alpha_{(k-1) n}\left(T_{k-1}^{n} y_{(k-2) n}-p\right)\right\|+s_{n} \\
& \leq\left(1+r_{n}\right)\left[\left(1-\alpha_{(k-1) n}\right)\left\|y_{(k-2) n}-p\right\|+\alpha_{(k-1) n}\left\|T_{k-1}^{n} y_{(k-2) n}-p\right\|\right]+s_{n} \\
& \leq\left(1+r_{n}\right)\left[\left(1-\alpha_{(k-1) n}\right)\left\|y_{(k-2) n}-p\right\|+\alpha_{(k-1) n}\left(1+r_{n}\right)\left\|y_{(k-2) n}-p\right\|+\alpha_{(k-1) n} s_{n}\right]+s_{n} \\
& \leq\left(1+r_{n}\right)^{2}\left\|y_{(k-2) n}-p\right\|+s_{n}\left(1+r_{n}\right)+s_{n} \\
& \vdots \\
& \leq\left(1+r_{n}\right)^{k-i}\left\|y_{i n}-p\right\|+s_{n} \sum_{i=1}^{k-1}\left(1+r_{n}\right)^{k-i},
\end{aligned}
$$

for $i=1,2, \ldots, k-1$. It follows that

$$
c \leq \liminf _{n \rightarrow \infty}\left\|y_{i n}-p\right\|, \quad \text { for } i=1,2, \ldots, k-1 .
$$

From (4.25) and (4.27), we obtain

$$
\lim _{n \rightarrow \infty}\left\|y_{\text {in }}-p\right\|=c, \quad \text { for } i=1,2, \ldots, k-1,
$$

and then

$$
\lim _{n \rightarrow \infty}\left\|\left(1-\alpha_{i n}\right)\left(y_{(i-1) n}-p\right)+\alpha_{i n}\left(T_{i}^{n} y_{(i-1) n}-p\right)\right\|=c,
$$

for $i=1,2, \ldots, k-1$.

Since $\left\|T_{i}^{n} y_{(i-1) n}-p\right\| \leq\left(1+r_{n}\right)\left\|y_{(i-1) n}-p\right\|+s_{n}$, for $i=1,2, \ldots, k-1$, we have

$$
\limsup _{n \rightarrow \infty}\left\|T_{i}^{n} y_{(i-1) n}-p\right\| \leq c, \quad \text { for } i=1,2, \ldots, k-1
$$


From (4.25), (4.29), (4.30) and Lemma 2.2, we obtain

$$
\lim _{n \rightarrow \infty}\left\|T_{i}^{n} y_{(i-1) n}-y_{(i-1) n}\right\|=0, \quad \text { for } i=1,2, \ldots, k-1
$$

Now we want to show that (4.31) is also true for $i=k$.

Since $\left\|T_{k}^{n} y_{(k-1) n}-p\right\| \leq\left(1+r_{n}\right)\left\|y_{(k-1) n}-p\right\|+s_{n}, \lim _{n \rightarrow \infty} r_{n}=0$ and $\lim _{n \rightarrow \infty} s_{n}=0$, it follows by (4.28),

$$
\limsup _{n \rightarrow \infty}\left\|T_{k}^{n} y_{(k-1) n}-p\right\| \leq c
$$

We also have

$$
\lim _{n \rightarrow \infty}\left\|\left(1-\alpha_{k n}\right)\left(y_{(k-1) n}-p\right)+\alpha_{k n}\left(T_{k}^{n} y_{(k-1) n}-p\right)\right\|=\lim _{n \rightarrow \infty}\left\|x_{n+1}-p\right\|=c .
$$

Hence, by (4.25), (4.32), and Lemma 2.2, we obtain

$$
\lim _{n \rightarrow \infty}\left\|y_{(k-1) n}-T_{k}^{n} y_{(k-1) n}\right\|=0
$$

Then, (4.31) and (4.34) give us

$$
\lim _{n \rightarrow \infty}\left\|T_{i}^{n} y_{(i-1) n}-y_{(i-1) n}\right\|=0, \quad \text { for } i=1,2, \ldots, k
$$

From

$$
\left\|x_{n}-T_{i}^{n} y_{(i-1) n}\right\| \leq\left\|x_{n}-y_{(i-1) n}\right\|+\left\|y_{(i-1) n}-T_{i}^{n} y_{(i-1) n}\right\|,
$$

it implies by (4.15) and (4.35) that

$$
\lim _{n \rightarrow \infty}\left\|x_{n}-T_{i}^{n} y_{(i-1) n}\right\|=0,
$$

for $i=1,2,3, \ldots, k$.

(ii) From part (i), for $i=1$, we have

$$
\lim _{n \rightarrow \infty}\left\|T_{1}^{n} x_{n}-x_{n}\right\|=0 .
$$

For $i=2,3,4, \ldots, k$, we get

$$
\begin{aligned}
\left\|T_{i}^{n} x_{n}-x_{n}\right\| & \leq\left\|T_{i}^{n} x_{n}-T_{i}^{n} y_{(i-1) n}\right\|+\left\|T_{i}^{n} y_{(i-1) n}-x_{n}\right\| \\
& \leq L\left\|x_{n}-y_{(i-1) n}\right\|^{\gamma^{i}}+\left\|T_{i}^{n} y_{(i-1) n}-x_{n}\right\| .
\end{aligned}
$$


By part (i) and (4.15),

$$
\lim _{n \rightarrow \infty}\left\|T_{i}^{n} x_{n}-x_{n}\right\|=0, \quad \text { for } i=1,2, \ldots, k \text {. }
$$

For $1 \leq i \leq k$, we obtain

$$
\begin{aligned}
\left\|x_{n}-T_{i} x_{n}\right\| \leq & \left\|x_{n}-x_{n+1}\right\|+\left\|x_{n+1}-T_{i}^{n+1} x_{n+1}\right\| \\
& +\left\|T_{i}^{n+1} x_{n+1}-T_{i}^{n+1} x_{n}\right\|+\left\|T_{i}^{n+1} x_{n}-T_{i} x_{n}\right\| \\
\leq & \left\|x_{n}-x_{n+1}\right\|+\left\|x_{n+1}-T_{i}^{n+1} x_{n+1}\right\| \\
& +L\left\|x_{n+1}-x_{n}\right\|^{\gamma_{i}}+L\left\|T_{i}^{n} x_{n}-x_{n}\right\|^{\gamma_{i}} .
\end{aligned}
$$

From (4.19) and (4.40), we then have

$$
\lim _{n \rightarrow \infty}\left\|x_{n}-T_{i} x_{n}\right\|=0, \quad \text { for } i=1,2, \ldots, k
$$

Theorem 4.3. Under the hypotheses of Lemma 4.2, assume that $T_{j}^{m}$ is semicompact for some positive integers $m$ and $1 \leq j \leq k$. Then $\left\{x_{n}\right\}$ converges strongly to a common fixed point of the family $\left\{T_{i}: i=1,2, \ldots, k\right\}$.

Proof. Suppose that $T_{j}^{m}$ is semicompact for some positive integers $m \geq 1$ and $1 \leq j \leq k$. We have

$$
\begin{aligned}
\left\|T_{j}^{m} x_{n}-x_{n}\right\| \leq & \left\|T_{j}^{m} x_{n}-T_{j}^{m-1} x_{n}\right\|+\left\|T_{j}^{m-1} x_{n}-T_{j}^{m-2} x_{n}\right\| \\
& +\cdots+\left\|T_{j}^{2} x_{n}-T_{j} x_{n}\right\|+\left\|T_{j} x_{n}-x_{n}\right\| \\
\leq & (m-1) L\left\|T_{j} x_{n}-x_{n}\right\|^{\gamma_{j}}+\left\|T_{j} x_{n}-x_{n}\right\| .
\end{aligned}
$$

Then, by Lemma 4.2(ii), we get $\left\|T_{j}^{m} x_{n}-x_{n}\right\| \rightarrow 0$ as $n \rightarrow \infty$. Since $\left\{x_{n}\right\}$ is bounded and $T_{j}^{m}$ is semicompact, there exists a subsequence $\left\{x_{n_{l}}\right\}$ of $\left\{x_{n}\right\}$ such that $x_{n_{l}} \rightarrow q \in C$ as $l \rightarrow \infty$.

By continuity of $T_{i}$ and Lemma 4.2(ii), we obtain

$$
\left\|q-T_{j} q\right\|=\lim _{l \rightarrow \infty}\left\|x_{n_{l}}-T_{j} x_{n_{l}}\right\|=0, \quad \forall j=1,2, \ldots, k
$$

Therefore, $q \in F$ and then Theorem 3.2 implies that $\left\{x_{n}\right\}$ converges strongly to a common fixed point $q$ of the family $\left\{T_{i}: i=1,2, \ldots, k\right\}$.

We note that in practical Theorem 4.3 is very useful in the case that one of $T_{i}, i=$ $1,2,3, \ldots, k$, is semicompact.

Theorem 4.4. Let $C$ be a nonempty closed convex subset of an uniformly convex real Banach space $X$ satisfying the Opial property, and $\left\{T_{i}: i=1,2, \ldots, k\right\}$ be a family of $\left(L-\gamma_{i}\right)$ uniform Lipschitz and 
generalized asymtotically quasinonexpansive self-mappings of $C$, that is, $\left\|T_{i}^{n} x-T_{i}^{n} y\right\| \leq L\|x-y\|^{\gamma_{i}}$ and $\left\|T_{i}^{n} x-p_{i}\right\| \leq\left(1+r_{i n}\right)\left\|x-p_{i}\right\|+s_{i n}$, for all $x, y \in C$ and $p_{i} \in F\left(T_{i}\right), i=1,2, \ldots, k$. Suppose that $F=$ $\bigcap_{i=1}^{k} F\left(T_{i}\right) \neq \emptyset$. Let $x_{1} \in C$ and the iterative sequence $\left\{x_{n}\right\}$ be defined by (1.2) with $\left\{\alpha_{i n}\right\}_{i=1}^{n} \subset[a, b]$, where $0<a<b<1$. Assume that $\sum_{n=1}^{\infty} r_{n}<\infty$, where $r_{n}=\max _{1 \leq i \leq k}\left\{r_{i n}\right\}$. If $I-T_{i}, i=1,2, \ldots, k$, is demiclosed at 0 , then $\left\{x_{n}\right\}$ converges weakly to a common fixed point of the family of mappings.

Proof. Let $p \in F$. By Lemma 3.1(v), we get $\lim _{n \rightarrow \infty}\left\|x_{n}-p\right\|$ exists. Then we follow the proof of Theorem 3.2 by Khan et al. [1] until we can conclude that $\left\{x_{n}\right\}$ converges weakly to a common fixed point $p \in F$.

\section{Acknowledgments}

This paper was supported by grant from under the program Strategic Scholarships for Frontier Research Network for the Ph. D. Program Thai Doctoral degree from the Office of the Higher Education Commission, the Graduate School of Chiang Mai University, and the Thailand Research Fund.

\section{References}

[1] A. R. Khan, A.-A. Domlo, and H. Fukhar-ud-din, "Common fixed points Noor iteration for a finite family of asymptotically quasi-nonexpansive mappings in Banach spaces," Journal of Mathematical Analysis and Applications, vol. 341, no. 1, pp. 1-11, 2008.

[2] J. Schu, "Weak and strong convergence to fixed points of asymptotically nonexpansive mappings," Bulletin of the Australian Mathematical Society, vol. 43, no. 1, pp. 153-159, 1991.

[3] K.-K. Tan and H. K. Xu, "Fixed point iteration processes for asymptotically nonexpansive mappings," Proceedings of the American Mathematical Society, vol. 122, no. 3, pp. 733-739, 1994.

[4] B. Xu and M. A. Noor, "Fixed-point iterations for asymptotically nonexpansive mappings in Banach spaces," Journal of Mathematical Analysis and Applications, vol. 267, no. 2, pp. 444-453, 2002.

[5] A. Kettapun, A. Kananthai, and S. Suantai, "A new approximation method for common fixed points of a finite family of asymptotically quasi-nonexpansive mappings in Banach spaces," Computers and Mathematics with Applications, vol. 60, no. 5, pp. 1430-1439, 2010.

[6] S. Imnang and S. Suantai, "Common fixed points of multistep Noor iterations with errors for a finite family of generalized asymptotically quasi-nonexpansive mappings," Abstract and Applied Analysis, vol. 2009, Article ID 728510, 14 pages, 2009.

[7] S. Saejung, S. Suantai, and P. Yodkaew, "A note on "Common fixed points of multistep Noor iterations with errors for a finite family of generalized asymptotically quasi-nonexpansive mappings"," Abstract and Applied Analysis, vol. 2009, Article ID 283461, 9 pages, 2009.

[8] S. H. Khan and W. Takahashi, "Approximating common fixed points of two asymptotically nonexpansive mappings," Scientiae Mathematicae Japonicae, vol. 53, no. 1, pp. 143-148, 2001.

[9] L.-C. Zeng and J.-C. Yao, "Strong convergence theorem by an extragradient method for fixed point problems and variational inequality problems," Taiwanese Journal of Mathematics, vol. 10, no. 5, pp. 1293-1303, 2006.

[10] Y.-C. Lin, N.-C. Wong, and J.-C. Yao, "Strong convergence theorems of Ishikawa iteration process with errors for fixed points of Lipschitz continuous mappings in Banach spaces," Taiwanese Journal of Mathematics, vol. 10, no. 2, pp. 543-552, 2006.

[11] L.-C. Zeng and J.-C. Yao, "Stability of iterative procedures with errors for approximating common fixed points of a couple of $q$-contractive-like mappings in Banach spaces," Journal of Mathematical Analysis and Applications, vol. 321, no. 2, pp. 661-674, 2006.

[12] L.-C. Zeng and J.-C. Yao, "Implicit iteration scheme with perturbed mapping for common fixed points of a finite family of nonexpansive mappings," Nonlinear Analysis: Theory, Methods $\mathcal{E}$ Applications, vol. 64, no. 11, pp. 2507-2515, 2006.

[13] L. C. Ceng, P. Cubiotti, and J. C. Yao, "Approximation of common fixed points of families of nonexpansive mappings," Taiwanese Journal of Mathematics, vol. 12, no. 2, pp. 487-500, 2008. 
[14] L. C. Ceng, P. Cubiotti, and J. C. Yao, "Strong convergence theorems for finitely many nonexpansive mappings and applications," Nonlinear Analysis: Theory, Methods E Applications, vol. 67, no. 5, pp. 1464-1473, 2007.

[15] Y. Yao, J.-C. Yao, and H. Zhou, "Approximation methods for common fixed points of infinite countable family of nonexpansive mappings," Computers $\mathcal{E}$ Mathematics with Applications, vol. 53, no. 9, pp. 1380-1389, 2007.

[16] A. Petruşel and J.-C. Yao, "Viscosity approximation to common fixed points of families of nonexpansive mappings with generalized contractions mappings," Nonlinear Analysis: Theory, Methods \& Applications, vol. 69, no. 4, pp. 1100-1111, 2008.

[17] Z.-h. Sun, "Strong convergence of an implicit iteration process for a finite family of asymptotically quasi-nonexpansive mappings," Journal of Mathematical Analysis and Applications, vol. 286, no. 1, pp. 351-358, 2003.

[18] N. Shahzad and A. Udomene, "Approximating common fixed points of two asymptotically quasinonexpansive mappings in Banach spaces," Fixed Point Theory and Applications, vol. 2006, Article ID 18909, 10 pages, 2006.

[19] N. Shahzad and H. Zegeye, "Strong convergence of an implicit iteration process for a finite family of generalized asymptotically quasi-nonexpansive maps," Applied Mathematics and Computation, vol. 189, no. 2, pp. 1058-1065, 2007.

[20] L.-C. Ceng, H.-K. Xu, and J.-C. Yao, "The viscosity approximation method for asymptotically nonexpansive mappings in Banach spaces," Nonlinear Analysis: Theory, Methods $\mathcal{E}$ Applications, vol. 69, no. 4, pp. 1402-1412, 2008.

[21] L.-C. Ceng, H.-K. Xu, and J.-C. Yao, "Strong convergence of an iterative method with perturbed mappings for nonexpansive and accretive operators," Numerical Functional Analysis and Optimization, vol. 29, no. 3-4, pp. 324-345, 2008.

[22] H.-K. Xu and R. G. Ori, "An implicit iteration process for nonexpansive mappings," Numerical Functional Analysis and Optimization, vol. 22, no. 5-6, pp. 767-773, 2001.

[23] K. Nammanee, M. A. Noor, and S. Suantai, "Convergence criteria of modified Noor iterations with errors for asymptotically nonexpansive mappings," Journal of Mathematical Analysis and Applications, vol. 314, no. 1, pp. 320-334, 2006.

[24] H. Fukhar-ud-din and A. R. Khan, "Approximating common fixed points of asymptotically nonexpansive maps in uniformly convex Banach spaces," Computers $\mathcal{E}$ Mathematics with Applications, vol. 53, no. 9, pp. 1349-1360, 2007.

[25] K.-K. Tan and H. K. Xu, "Approximating fixed points of nonexpansive mappings by the Ishikawa iteration process," Journal of Mathematical Analysis and Applications, vol. 178, no. 2, pp. 301-308, 1993.

[26] H. Fukhar-ud-din and S. H. Khan, "Convergence of iterates with errors of asymptotically quasinonexpansive mappings and applications," Journal of Mathematical Analysis and Applications, vol. 328, no. 2, pp. 821-829, 2007. 


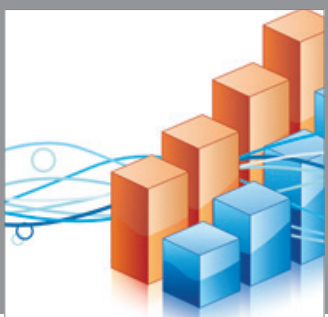

Advances in

Operations Research

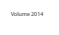

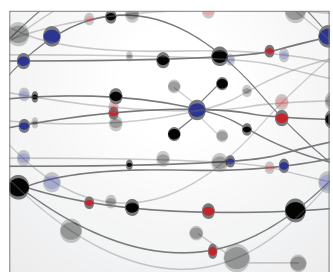

\section{The Scientific} World Journal
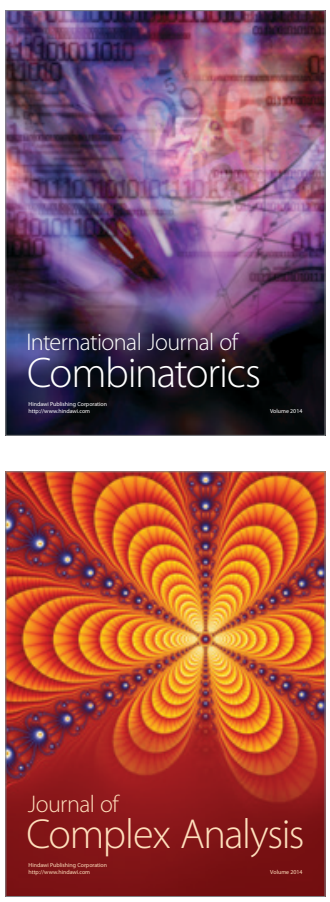

International Journal of

Mathematics and

Mathematical

Sciences
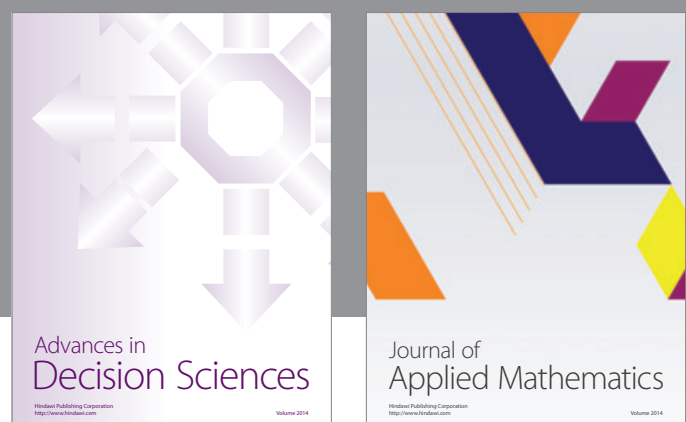

Journal of

Applied Mathematics
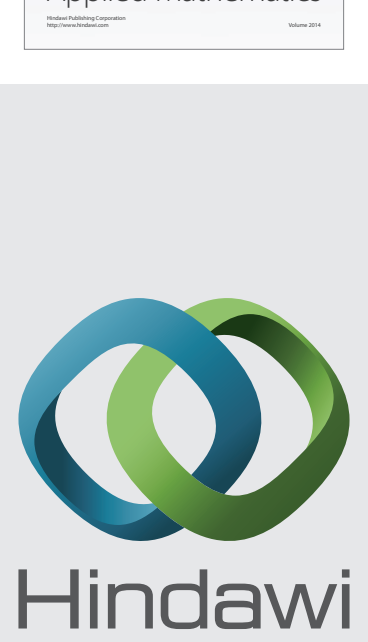

Submit your manuscripts at http://www.hindawi.com
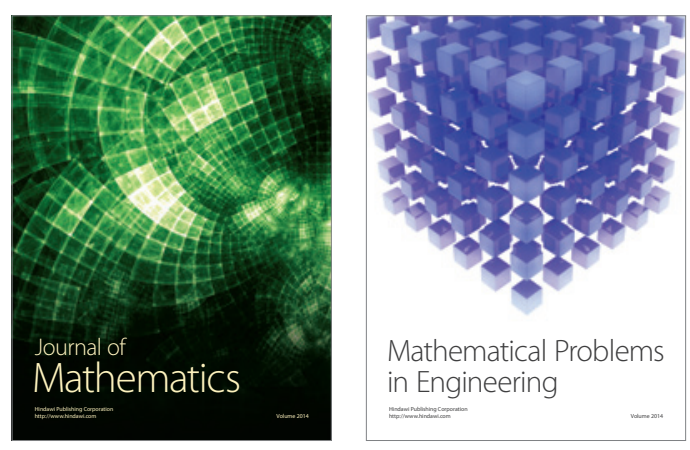

Mathematical Problems in Engineering
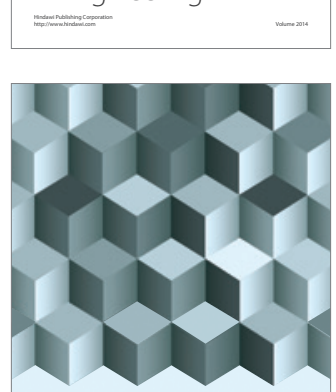

Journal of

Function Spaces
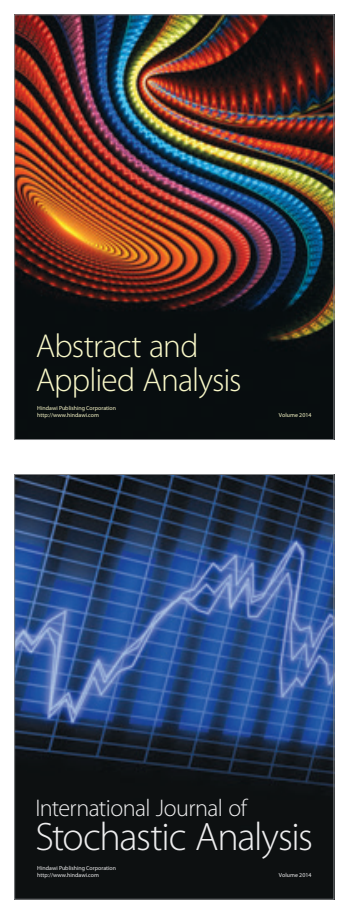

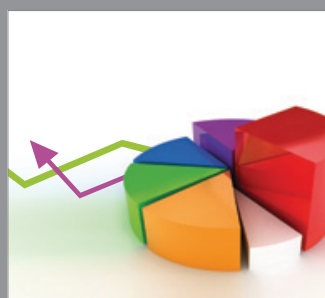

ournal of

Probability and Statistics

Promensencen
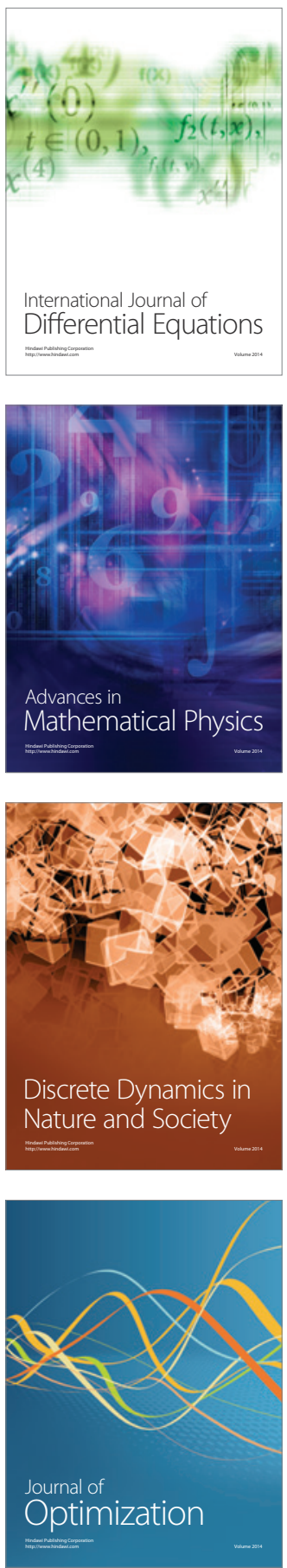